\title{
Distribution of the Infection Time of Ascospores of Venturia inaequalis
}

\author{
Vincent Philion, ${ }^{1, \dagger}$ Valentin Joubert, ${ }^{1}$ Marc Trapman, ${ }^{2}$ Anne-Grete R. Hjelkrem, ${ }^{3}$ and Arne Stensvand, ${ }^{4,5}$ \\ ${ }^{1}$ Institut de Recherche et de Développement en Agroenvironnement, IRDA, 335 rang des Vingt-Cinq Est, Saint-Bruno-de- \\ Montarville, Quebec, Canada J3V 0G7 \\ ${ }^{2}$ Bio Fruit Advies, Dorpsstrat 32a, 4111 KT Zoelmond, The Netherlands \\ ${ }^{3}$ Division of Food Production and Society, Norwegian Institute of Bioeconomy Research, 1431 As, Norway \\ ${ }^{4}$ Division of Biotechnology and Plant Health, Norwegian Institute of Bioeconomy Research, 1431 As, Norway \\ ${ }^{5}$ Department of Plant Science, Norwegian University of Life Sciences, 1433 Ås, Norway
}

\begin{abstract}
In many areas where spring is wet, fungicides are applied in relation to rain events that trigger ejection of ascospores of Venturia inaequalis, which cause primary infections of apple scab. Past studies established the rate of ejection during rain in relation to light and temperature, and determined the wetting time required for infection. Simulation software uses this information to calculate risk and help time sprays accordingly. However, the distribution of the infection time required by a population of spores landed on leaves was never studied, and assumptions were

were enumerated after incubation. Lesions increased with wetness time and leveled off once the slowest spores infected the host, closely matching the monomolecular model. Wetness hours were best adjusted for temperature using the Yin equation. The minimum infection time on the youngest leaves was about $5 \mathrm{~h}$, matching results from previous studies, whereas half the lesions appeared after $7 \mathrm{~h}$ of infection. Infection times for leaves with ontogenic resistance were longer. Our results improve current software estimates and may improve spraying decisions.
\end{abstract} used. To estimate this, we inoculated ascospores of $V$. inaequalis on potted trees at different temperatures for specific wetting times. Lesions
Keywords: epidemiology, disease warning systems, fruit, tree fruits, fungi
In wet and cool apple growing regions around the world, apple scab caused by the ascomycete Venturia inaequalis is the most important disease, and it dictates plant protection measures. Although conidial inoculum may be present in twigs and buds of apple in poorly managed orchards, ascospores produced in fruiting bodies (pseudothecia) in leaf litter on the ground is the predominant primary inoculum of $V$. inaequalis.

The infection requirements for ascospores of this pathogen have been studied for close to a century. A popular decision support system (DSS) was derived from these, with risk assessment curves that described the leaf wetness duration and temperature that resulted in light, medium, and severe infection (Mills 1944), and growers have successfully adapted their spraying schedules to account for the infection risk. There are numerous publications with refinements and revisions of this DSS. The light, moderate, and heavy infection curves stated by Mills (1944) were defined by Schwabe (1980) as $<10,10-40$, and $>40 \%$ scab developing on the foliage. However, these numbers are relative, because the maximum number of lesions may vary considerably with temperature (Schwabe 1980). Many studies published before and after Mills (Gadoury et al. 1996; Keitt and Jones 1926; MacHardy and Gadoury 1989; Moore 1964; Olivier et al. 1983; Preece 1964; Schwabe 1980; Stensvand et al. 1997; Sys and Soenen 1970) have suggested lower infection thresholds. Current risk assessments to a large extent use the minimal infection

\section{${ }^{\dagger}$ Corresponding author: V. Philion; vincent.philion@irda.qc.ca}

Funding: This project received financial support from the ministère de l'Agriculture, des Pêcheries et de l'Alimentation du Québec (MAPAQ), as part of the "Stratégie phytosanitaire québécoise en agriculture 2011-2021" under component 4 of the Prime-Vert 2013-2018 program. The work was also financially supported by the Research Council of Norway through the project SMARTCROP. The major apple forecast integration association coordinated the work.

The author(s) declare no conflict of interest.

Accepted for publication 19 August 2019.

(C) 2020 The American Phytopathological Society criteria as the spraying threshold for each rain event. However, for any rain the actual scab risk for a given orchard is not only a question of meeting the minimum wetness requirements for fungal infection, but also depends on the quantity of inoculum present (MacHardy et al. 1993), spore maturation level at the time of infection (Gadoury and MacHardy 1982), the ejection rate during rain (Stensvand et al. 1997), and the day and night periodicity as ejections are inhibited at night (Brook 1969; Gadoury et al. 1998). Once mature ascospores have ejected from the leaf litter and landed on the host, the distribution of the infection speed between the fastest and the slowest ascospores further spreads the infection process time and thus interacts with the scab risk since leaves may dry before all ejected spores can succeed in infecting the host.

Precise knowledge of the risk at any given moment during rain can help growers determine if previous sprays applied in relation to the infection event were sufficient, or if spore deposition on new growth or wash-off because of rain since the previous spray warrant an additional intervention. Providing such information to advisors and growers is the goal of decision support systems based on dynamic simulation such as RIMpro (Trapman and Polfliet 1997) and A-Scab (Rossi et al. 2007), which model each process (spore maturation, dissemination, and infection). However, the current implementation of these systems for the distribution of the speed of infection is based on the rate of ascospore germination and appressoria formation, and assumptions on how that translates to infection. Our objective was to establish the infection distribution curve for ascospores of $V$. inaequalis, so it can be directly integrated in decision support systems. Specifically, our goal was to model the proportion of ascospores that could infect leaves in relation to wetness duration, and a temperature dependent developmental rate.

\section{Materials and Methods}

Ascospore production. Scab infected leaves of apple cv. McIntosh were picked in late autumn from a heavily scabbed research orchard in Saint-Bruno-de-Montarville, Quebec. Leaves were incubated at $4{ }^{\circ} \mathrm{C}$ in the dark, with the adaxial surface facing upward, until mature pseudothecia were observed, usually after 16 weeks. Throughout the incubation period, leaves were misted with water $(5 \mathrm{~min} / \mathrm{h})$. Leaves bearing pseudothecia with ascospores apparently almost mature (James and Sutton 1982) (stage 11) were frozen until 
use. Leaves were placed in plastic wire screens, designed to fit custom-built ascospore harvesting tower spore traps similar to a previous design (Gilpatrick et al. 1972). On the days experiments were carried out, the screens were placed facing downward over the trap on a large section of funnels connected to a suction device. The traps were exposed to artificial light to ensure spore ejection (Brook 1969). The airflow (room temperature, $20-25^{\circ} \mathrm{C}$ ) in the traps was regulated with a flow meter and optimized such that spores naturally ejected from the wet leaves were impacted in a dry Eppendorf vial set directly under each funnel. Since a thin water film is required for ejection (MacHardy 1996), samples were rewetted every 20 min during the 2-h collection. Vials with ejected ascospores were temporarily maintained on ice. Just prior to tree inoculation, spores were resuspended in $200 \mu \mathrm{l}$ of water, and their concentration was evaluated with a hemacytometer and adjusted accordingly. Spore germination was not assessed for every run but averaged $91 \%$ across numerous experiments using the same technique. Between ejections, leaf samples were incubated in water-saturated air at $18^{\circ} \mathrm{C}$ to allow ascospore maturation but prevent discharge, or at $4^{\circ} \mathrm{C}$ in dry air to slow the process until the next trial.

Tree inoculation. Potted trees of apple cv. McIntosh grafted on different rootstocks were used in all experiments. Trees with one to three shoots were grown outside to ensure that leaves formed a cuticle comparable to what is found in commercial orchards but were sheltered during rain events to avoid natural infections of scab. Two to $3 \mathrm{~h}$ before each experimental run, the trees were acclimated at the randomly selected test temperature. Trees were sequentially inoculated, and immediately placed in a dark growth chamber equipped with mists (Conviron S10H, Controlled Environment Ltd., Winnipeg, Canada) to favor infection. During inoculation, an airbrush was used to precisely deposit $50 \mu \mathrm{l}$ of a fresh ascospore suspension $(0.5$ ascospores per $\mu l)$ on the adaxial surface of each of the three youngest completely unfurled leaves of at least one actively growing shoot per tree. These were labeled with increasing age as positions $0,-1$, and -2 . Groups of four trees were randomly removed from the infection chamber after fixed times, accounting for the inoculation delay. Leaves were quickly blotted with paper towels (Moore 1964) and air dried with a fan in ca. 5 min and immediately returned to a dry incubator at the test temperature until all trees were removed from the infection chamber. All trees were then moved to an incubation chamber (approximately $22^{\circ} \mathrm{C}$ and $70 \% \mathrm{RH}$ ) for 26-28 days to ensure complete disease expression on the inoculated leaves, accounting for ontogenic resistance (Keitt and Jones 1926). Powdery mildew was prevented with careful sanitation before tree inoculation, UV lighting in the air ducts of the growth chamber, and brief rewetting of leaves every 3-5 days during incubation. Finally, scab lesions were enumerated on each leaf. Since lesion density was 0 to 6 per leaf, discrete scab colonies were easily counted. The experiment was carried out as individual runs for each temperature $(4,5,6,8$, $11,14,18$, and $24^{\circ} \mathrm{C}$ ), and performed twice at 6,18 , and $24^{\circ} \mathrm{C}$; altogether 11 runs. Wetness times of up to $96 \mathrm{~h}$ were tested for temperatures below $14^{\circ} \mathrm{C}, 72 \mathrm{~h}$ for $18^{\circ} \mathrm{C}$, and $48 \mathrm{~h}$ for $24^{\circ} \mathrm{C}$.

Infection model. The effect of wetness duration on scab severity was modeled as a nonlinear least square (nls) using a monomolecular (also known as the negative exponential, von Bertalanffy, or Mitscherlich) function (Madden et al. 2007) with the addition of a horizontal shift (Equation 1).

$$
Y=Y_{\max } \cdot\left(1-e^{-r \cdot\left(W-W_{\min }\right)}\right)
$$

$Y$ is the average number of scab lesions per leaf (disease severity), described as a function of wetness duration $(W)$. The model contains three parameters, where $Y_{\max }$ is the maximum mean scab severity (asymptote), $r$ is the rate, and $W_{\min }$ is the minimum wetness duration required for infection, which serves as the horizontal location parameter (Bolker 2008). $Y$ was forced to zero during fitting for values of $W$ below $W_{\min }$. The nonlinear fit was done for each temperature run independently, with the number of scab lesions of all inoculated leaves per tree pooled and averaged per run and infection duration. The coefficients of the individual monomolecular curves (Equation 1) for each run were used to estimate the time required for $50 \%$ infection $\left(W_{50}\right)$ (Equation 2 ) and similarly for $95 \%$ infection.

$$
W_{50}=-\log (0.5) / r+W_{\min }
$$

The rate (reciprocal of the time) to reach minimum infection $\left(r_{\min }=\right.$ $\left.1 / W_{\min }\right)$ and $50 \%$ infection $\left(r_{50}=1 / W_{50}\right)$ was calculated for each temperature run and used to compare the suitability of timetemperature dependent models (Scherm and van Bruggen 1994).

Developmental rates. The effect of temperature on the calculated rates $r_{\min }$ and $r_{50}$ obtained above was explored with different models using nls. In the Logan-Lactin (Lactin et al. 1995) function (Equation $3)$, the rate $(r)$ is described as a function of temperature $(T)$. The model consists of three model parameters, where $p$ is the rate parameter, $T_{\max }$ is the maximum temperature, and $d$ is the width of the high temperature boundary.

$$
r=e^{p \cdot T}-e^{p \cdot T_{\max }-\left(\left(T_{\max }-T\right) / d\right)}
$$

In the Yin (Yan and Hunt 1999; Yin et al. 1995) function (Equation 4), the rate $(r)$ is described as a function of temperature $(T)$. The model contains four model parameters, where $a$ is the upper asymptote parameter (the rate at the optimal temperature), $T_{\max }$ is the maximum temperature, $T_{\text {min }}$ is the minimum temperature, and $T_{o p t}$ is the optimum temperature.

$$
r=a \cdot\left(\frac{\left(T_{\max }-T\right)}{\left(T_{\max }-T_{o p t}\right)}\right) \cdot\left(\frac{\left(T-T_{\min }\right)}{\left(T_{o p t}-T_{\min }\right)}\right)^{\left(\frac{\left(T_{o p t}-T_{\min }\right)}{\left(T_{\max }-T_{o p t}\right)}\right)}
$$

In addition to the Logan-Lactin (Equation 3) and the Yin (Equation 4) function, the linear (degree-hours), Brière (Brière et al. 1999), and finally two equations used by Damos and SavopoulouSoultani (2012); a function based on a simplified $\beta$ type distribution, and an inverse second-order polynomial were fitted. Realistic constraints were needed for model convergence for some functions.

The six rate functions were tested with both $r_{\min }$ and $r_{50}$ derived from our experimental data and additionally for $r_{\min }$ obtained from previous studies (Keitt and Jones 1926; Moore 1964; Preece 1964; Schwabe 1980; Stensvand et al. 1997; Sys and Soenen 1970; Tosic and Vasic 1977), some of which was reproduced from MacHardy and Gadoury (1989). We combined compiled results and original observations when available for each temperature. Both laboratory and orchard data from these studies were included despite the potential influence and uncertainty of data from varying outside temperatures. The pooled data of all studies including our own was fit both using a nonlinear generalized least squares model (gnls) and with a mixed effect model (nlme). For the latter, a simple approach was applied, attributing a random intercept for the maximum rate per study, assuming the variations between studies were the result of sampling error and that the same model applied to all datasets.

Infection models with pooled data. Scab severity of the whole dataset (leaf scab pooled and averaged per run and leaf position) was modeled again using Equation 1, but using an adjusted wet thermal time $\left(W_{a d j}\right)$ (Equation 5) instead of $W$ to account for the different temperatures.

The value of $W_{a d j}$ was obtained by fitting Equations 1 and 4 simultaneously, by multiplying the wetness duration $(W)$ with the developmental rate $(r)$ divided by the rate at the optimum temperature $(a)$ (both from Equation 4), thus scaling the rate at the optimum temperature to one. This was done to facilitate interpretation in terms of hours of leaf wetness.

$$
W_{a d j}=W \cdot r / a
$$

The pooled data were fit both using gnls and nlme. For the mixed models, different combinations of random effects accounting for the variations between runs, and leaf positions nested within runs, were compared for each fixed effect $\left(Y_{\max }, r, W_{\min }, T_{o p t}, T_{\max }\right.$, and $\left.T_{\min }\right)$. Individual leaf, shoot, and tree data were pooled and the mean was 
used to avoid addressing nonlinear models with a discrete response variable with an overly complex random structure.

Data analysis and software. The $\mathrm{R}$ language was used to handle data using the "tidyverse" packages (Wickham 2017), and to fit all models. The "nls" function from the "stats" package (R Core Team 2018) with the netlib port algorithm (n2lsol) was used to fit the infection model for individual temperatures and the developmental rate equations. The library nlstools (Baty et al. 2015) was used to evaluate the models. The $95 \%$ confidence intervals [presented in brackets] for the estimates of model parameters were calculated with a Taylor expansion and Monte Carlo simulation (Spiess 2018) and using "confint2" from the nlstools package (Baty et al. 2015) for the simple model coefficients. The more complex models with pooled data that involved covariates were explored with "gnls" (Pinheiro and Bates 2004) and with "nlme" (Pinheiro et al. 2018).

Model evaluation. Nonlinear model fitting was done following the approach described in Archontoulis and Miguez (2015). For the developmental rate models, only equations with parameter estimates within a reasonable range in relation to their biological interpretation were used. Models were selected based on the Akaike Information Criterion (AIC) and root mean square error (RMSE). The model with the lowest AIC and RMSE was regarded as the best model. If the difference in AIC ( $\triangle \mathrm{AIC}$ ) between two models was less than 2, the two models were considered to have an equally good fit. For the pooled data models, the distribution and independence of errors and homogeneous variance (homoscedasticity) were evaluated. Different combinations of nested fixed, random effects, and variance models were tested by comparing the model with and without the effect tested using $\triangle \mathrm{AIC}$ and likelihood ratios (LR) tests for which the $\chi^{2}$ statistics $\left(\chi^{2}\right)$, difference in the number of parameters for the two models (degrees of freedom), and associated $P$ values were reported. Mixed model performance was estimated with the concordance correlation coefficient (CCC), the coefficient of residual mass (CRM), and RMSE. CCC is the product of the Pearson correlation coefficient and $\mathrm{C}_{\mathrm{b}}$, an indicator of the difference between the best fitting line and the perfect agreement line (Madden et al. 2007), whereas CRM is a measure of the tendency of the model to overestimate or underestimate the observed values (Nash and Sutcliffe 1970).

\section{Results}

Infection model. For all experimental runs, scab lesions developed during incubation on the inoculated leaves only. Beyond an initial minimum wetness time, the number of lesions increased with increasing wetness duration, before it leveled off to an asymptote. The relationship between the average lesion number and wetness duration for each temperature closely followed the monomolecular function (Fig. 1). The minimum infection time varied with temperature, and there was a slight decrease in the asymptote with increasing temperature. The index of dispersion of raw counts at the leaf scale (variance to mean ratio) (Madden et al. 2007) did not reveal departure from a random Poisson process $(n=1,864, \mathrm{D}=0.9)$. For most runs, residual errors showed an increasing trend with increasing wetness duration. Variance heterogeneity was not modeled at this stage, since only the derived $r_{\min }$ and $r_{50}$ coefficients of each run were used to compare temperature rate equations.

Developmental rates. The plot of rates against temperature (Fig. 2) resulted in negatively skewed unimodal curves for both $r_{\min }$ and $r_{50}$, but the shape of the curve varied for the two rates and between data sets. For $r_{\min }$, the Logan-Lactin function (Equation 4) was selected based on lowest AIC (-70) and RMSE (0.007). The model parameters were estimated at $p=0.2015[0.188,0.215], T_{\max }=25.5^{\circ} \mathrm{C}$ $[25.3,25.8]$, and $d=4.94$ [4.6, 5.3]. The optimum temperature for minimum infection was derived from the function $\left(T_{o p t}=T_{\max }-d\right)$ and found to be $20.6^{\circ} \mathrm{C}$ [20.0, 21.2]. The predicted minimum infection time at the optimum temperature based on this curve was $3.5 \mathrm{~h}$ $[1.8,3.6]$. The tail of low values reflected lack of data at temperatures much higher than the optimal. The extrapolated rate at $0^{\circ} \mathrm{C}(0.023$ $[0.022,0.043])$ was about 12 times slower than the rate predicted at the optimal temperature. Parameter estimates were given with 4 digits, because similar to what was reported by Lactin et al.
(1995), we observed that the curve predictions were very sensitive to rounding error. Although the Damos inverse polynomial gave a similar fit (AIC $=-69$ ), the Logan-Lactin was preferred because it had a lower RMSE and the parameters have a direct biological interpretation. The Yin rate function with a parameter fit for minimal temperature also gave a nice fit $($ AIC $=-67$ ), but there was a big standard error for this parameter. The simpler model with minimum temperature fixed at $0^{\circ} \mathrm{C}$ had a much higher AIC (-50) and RMSE (0.018). The Brière, simplified beta Damos equations, and degree-days gave a poor fit to the data compared with other models (AIC > -51).

The $r_{50}$ curve was nearly symmetrical (Fig. 2), and the Yin rate function simplified by fixing $T_{\min }$ at $0^{\circ} \mathrm{C}$ (Equation 5) was selected $(\mathrm{AIC}=-75, \mathrm{RMSE}=0.006)$. The model parameters were estimated at $a=0.124[0.116,0.132], T_{\max }=34.8^{\circ} \mathrm{C}[30.0,39.7]$ and $T_{o p t}=$ $20.1^{\circ} \mathrm{C}[18.4,21.7]$. The rate is set to 0 for temperatures above $T_{\max }$ and below $T_{\min }$. However, the Damos simplified beta (AIC $=-75$, RMSE $=0.006$ ) and Brière rate functions (AIC $=-74$, RMSE $=$ 0.005 ) had similar fits. The Yin function was preferred to the Damos function because we could more easily extract cardinal temperatures. The Brière function had convergence issues, the fitted $T_{\max }$ did not have biological sense $\left(>80^{\circ} \mathrm{C}\right)$, and the standard errors of the coefficients were larger. The Logan-Lactin and linear degree-days had a poor fit compared with the other models (AIC > -63, RMSE > 0.009).

For the data obtained from previous studies, we could only reliably analyze minimum infection times and derive $r_{\min }$. Again, the fit of the Logan-Lactin function was not as good $($ AIC $=-354$, RMSE $=$ 0.022 ), and the Yin function was selected (AIC $=-365$, RMSE $=$ 0.021) (Equation 5, Fig. 2). Residuals increased with fitted values, and so heteroscedasticity was modeled with a simple exponential variance function (varExp) $\left(\chi^{2}=78(1), P<0.0001\right)$. Attributing a random intercept per study for the optimal rate (a) (gnls versus nlme) also improved the model $\left(\chi^{2}=5.5(1), P=0.02\right)$. The parameter estimates were: $a=0.201[0.181,0.221] ; T_{\max }=27.9^{\circ} \mathrm{C}[27.4,28.4]$; $T_{\text {opt }}=20.1^{\circ} \mathrm{C}[19.6,20.6]$, and $T_{\min }=-15.6^{\circ} \mathrm{C}[-24.9,-6.2]$.

Infection models with pooled data. Similar to model fits from individual runs, residuals increased with fitted values. This was expected because the response variable is the mean of a count (scab lesions). Heteroscedasticity was addressed with a simple exponential variance function (varExp) $\left(\chi^{2}=86(1), P<0.0001\right)$. By comparing models generated with gnls, we observed that the asymptote $\left(Y_{\max }\right)$ and the minimum time for infection $\left(W_{\min }\right)$ varied significantly between the three leaf positions $\left(\chi^{2}=185(2), P<0.0001\right.$ and $\chi^{2}=$ $37(2), P<0.0001$, respectively), while the rate $(r)$, optimal and max temperature $\left(T_{o p t}\right.$ and $\left.T_{\text {max }}\right)$ did not $\left(\chi^{2}<0.7(2), P>0.5\right)$. The asymptote was linearly significantly reduced with increasing temperature $\left(\chi^{2}=23(1), P<0.0001\right)$, but the effect was only apparent for the oldest leaves (interaction $\chi^{2}=9(2), P=0.01$ ). Use of a mixed model (nlme) further improved the model. Starting with the gnls model, a random intercept was added to account for variations in minimum infection time between runs $\left(\chi^{2}=27(1), P<0.001\right)$, which was expected because of the fixed time interval between tree removal from the chambers. Similarly, letting asymptote vary randomly per position within runs improved the model $\left(\chi^{2}=17(1), P<0.001\right)$, but a separate asymptote effect at the run level did not. Random intercept for rate per run and/or leaf position did not improve the model. The same fixed effects with similar coefficients were retained once the random effects were accounted for (gnls versus nlme), except the reduction of scab severity with increasing temperature for older leaves was a bit weaker with nlme $\left(0.02\right.$ lesion per ${ }^{\circ} \mathrm{C}, \Delta \mathrm{AIC}=$ $\left.6, \chi^{2}=12(3), P=0.009\right)$.

One outlier value (one leaf of $n=1865$ before averaging) was removed from the initial analysis. Visual inspection of residual plots of the model ( $n=312$ values) did not reveal any obvious deviations from homoscedasticity between runs, leaf position, and for each individual temperature. Model diagnostics revealed two outliers which were not removed since they did not affect parameter estimates.

Although the normal plot of the within-group residuals did not indicate any violations from the assumption of normality (Pinheiro and 
Bates 2004), residuals were not normally distributed according to the Shapiro-Wilk test ( $\mathrm{W}=0.978, P<0.001$ ). However, formal tests of normality are known to be very sensitive to small deviations from normality for large samples (Kozak and Piepho 2018), and residuals of a simpler model using data pooled per run, but not by leaf position, did not violate the normality assumption of the test. Finally, normal plots of the best linear unbiased predictions (BLUP) for both the runs and the positions within runs random effects did not show evidence of deviance from the normality assumption, and a plot using the "pairs" method (Pinheiro and Bates 2004) showed the random effects were independent.

A summary of the fixed effects of the mixed model results can be found in Table 1 and represented in Figure 3. Since data from all temperatures is plotted on the same panel, the asymptote reduction effect of temperature on older leaves (visible by comparing panels in Figure 1) is not illustrated. At $20^{\circ} \mathrm{C}$, the model predicts older leaves (Position -2) would have about half the number of scab lesions observed on young leaves, whereas at $4^{\circ} \mathrm{C}$ older leaves are predicted
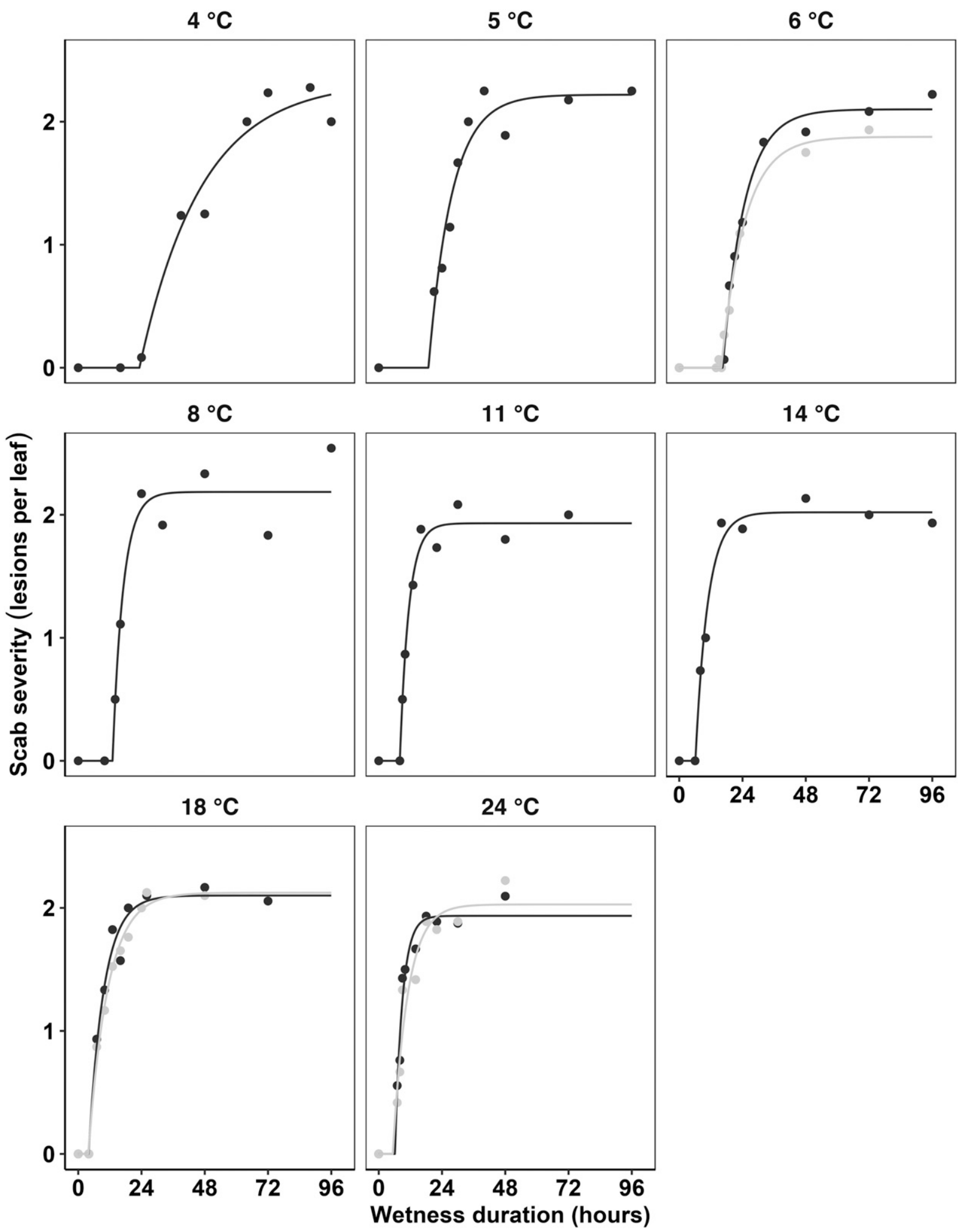

Fig. 1. Infection of apple leaves with ascospores of Venturia inaequalis in relation to leaf wetness duration for each individual temperature run. Average scab severity (Y) was modeled using Equation 1 for each run. Experiments at 6,18 , and $24^{\circ} \mathrm{C}$ were done twice. 
to have about $0.7 \times$ the scab of young leaves. Similarly, the infection time is expected on average 0.8 wetness hours (adjusted for temperature) lower for the youngest leaves compared with the oldest leaves.

Running the model with the Yin equation including $T_{\text {min }}$ (Equation 5) did not improve the model. The fitted $T_{\min }\left(-1.0^{\circ} \mathrm{C}\right)$ was not significantly different from $0^{\circ} \mathrm{C}\left(\chi^{2}=1.2(1), P=0.27\right)$ and the term was not included in the final model. As an alternative to simultaneously fitting $T_{o p t}$ and $T_{\max }$ alongside the other fixed effects, we used the cardinal temperatures obtained in the developmental rates section to calculate $W_{a d j}$ before fitting Equation 1 . The simultaneous fit of all parameters improved the model $\left(\chi^{2}=14.6(2), P<0.001\right.$ and $\left.\chi^{2}=5.2(2), P=0.08\right)$ compared with calculating $W_{a d j}$ from rates derived solely from $r_{\min }$ or $r_{50}$, respectively).

However, the "optimal" model selected using our data (Table 1) resulted in a minimum infection time at $2^{\circ} \mathrm{C}$ of $70 \mathrm{~h}, 35 \mathrm{~h}$ higher than the value suggested by Stensvand et al. (1997). Consequently, we explored the impact of tweaking the temperature rate function so that predictions at low temperatures matched this data point.

We compared the optimal model (m.0) to the model without forcing $T_{\text {min }}$ to $0^{\circ} \mathrm{C}$ (m.c), the models with forced cardinal values for $T_{o p t}$, $T_{\max }$, and $T_{\min }$ based on $r_{\min }$ (mOM.0) using the international data, or based on $r_{50}$ (mOM.50), and finally a model with a $T_{\min }$ low enough to predict infection within $32 \mathrm{~h}$ at $2^{\circ} \mathrm{C}(\mathrm{m} .18)$, the lowest values observed by Stensvand et al. (1997). The five models (Table 2) had similar values for asymptote $\left(Y_{\max }\right)$, effect of temperature on asymptote $\left(Y_{\max }: T\right)$, and had acceptable residuals. The concordance correlation coefficient (CCC) was the same for all; an overall value of 0.96 , with the precision component of 0.96 and accuracy $>0.99$. The only noticeable difference between variants was that the minimum time for infection $\left(W_{\min }\right)$ and infection rate $(r)$ were in relation to the cardinal temperature adjustment. The models with the heaviest weight on low temperatures had lower minimum infection time and higher infection rate values, whereas the model with more weight on high temperatures had longer predicted minimum infection time and lower infection rate values. The model with the best fit to our data $(\mathrm{m} 0)$ was intermediate. These differences slightly affected CRM and RMSE. All models had a small tendency to overestimate symptoms $(\mathrm{CRM}>0)$.

Since all models had similar performance, we chose the m.18 model variant to build the risk curve (Fig. 4) so that low temperature infections would not be missed and because it did not affect so much our estimate of wetness duration required for minimal, or $50 \%$ infection.

\section{Discussion}

As far as we know, this is the first successful quantification of the relationship between temperature, wetness duration, and infection efficiency of ascospores of $V$. inaequalis. The cumulative distribution of the infection time required by a population of simultaneously inoculated ascospores to result in visible lesions closely follows a monomolecular relation. We found that minimal wetness threshold and leaf wetness time required to reach $50 \%$ infection is lower than

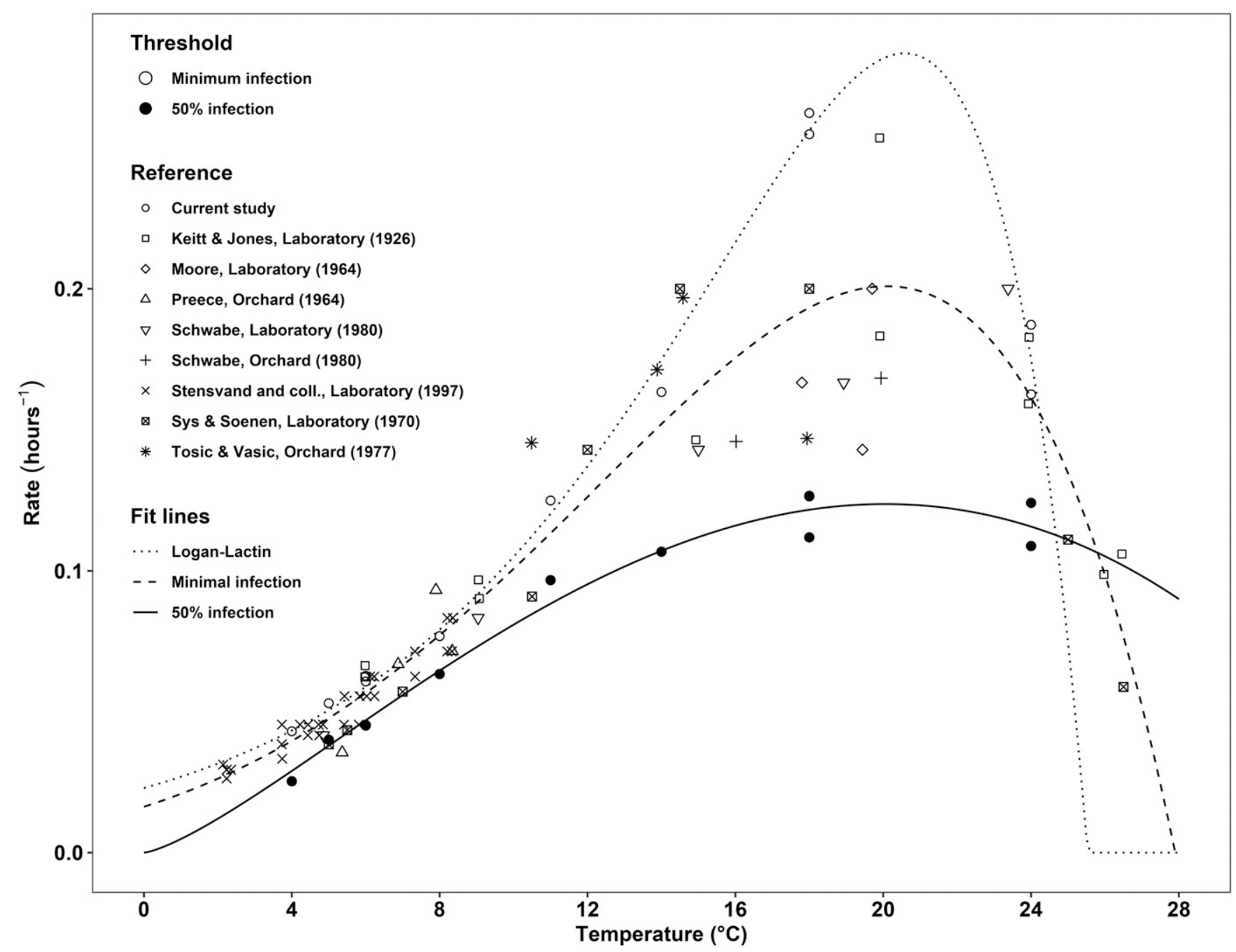

Fig. 2. Inverse of the wetness duration requirement following inoculation of ascospores of Venturia inaequalis for minimum and $50 \%$ infection on apple leaves. Rates were modeled (lines) using the Logan-Lactin function (Equation 3) for the minimal infection rate of the current study, whereas the rate of $50 \%$ infection and the pooled data for minimum infection from referenced studies were modeled using the Yin rate function (Equation 4). 
what is currently used in disease warning models for apple scab. The results also gave some insight into ontogenic resistance, and we are unaware of other studies reporting the increase in minimum wetness time required for infection with leaf age. This work permits to better model ascospore infection progress in relation to wetness duration and temperature. Once this new information is integrated in apple scab simulation models, it may enable a better comprehension of primary infection dynamics and more tailored spray decisions.

Although Schwabe (1980) had objectives very close to what is presented here and observed general trends of increasing severity with wetness duration, and slower infection rates at low temperatures, the infection pattern reported was somewhat erratic and could not be used in models. In a review of this work, MacHardy (1996) concluded it should be interpreted with caution, as a given amount of inoculum did not cause the same amount of infection between experiments. The uncontrolled inoculation technique used by Schwabe (1980) likely caused this.

This type of work was done for many pathogens with different models (Arauz and Sutton 1989; Butler and Jadhav 1991; Butler et al. 1994; Duthie 1997; Lalancette et al. 1988; Webb and Nutter 1997). A monomolecular cumulative distribution of the infection time as proposed in the present study is known to be a good candidate model when all spores (or seeds) have the same probability of germination per unit time (Brown and Mayer 1988). Our results also showed that the wetness duration can be adjusted for temperature with a rate, and similarly to Magarey et al. (2005), we also chose the Yin temperature response curve for both the minimum and $50 \%$ infection thresholds. Since the curve for minimum infection obtained with our data resulted in a different shape than the $50 \%$ curve, we decided to include data from other studies to confirm the observation. Since there was no evidence for such skew in the pooled results, we concluded that it was an artifact of methodology. It is likely the fixed timing for removing plants for the two $18^{\circ} \mathrm{C}$ runs resulted in both a lower predicted minimum infection time $\left(W_{\text {min }}\right)$, and thus a high rate $\left(r_{\text {min }}\right)$ for that temperature, which biased the minimum infection rate curve.

Although it is well recognized that younger leaves are more susceptible to apple scab than older leaves (MacHardy 1996), we were unfamiliar with an increase in minimum infection time with leaf age. The fact that leaf age impacts the lesion causing efficiency of ascospores by increasing the minimum time for infection, and not the rate at which subsequent spores realize infection, is compatible with the view that ontogenic resistance acts very early in the infection process and is more

Table 1. Summary of the mixed-effects nonlinear regression model of the impact of temperature and wetness duration on infection by ascospores of Venturia inaequalis of leaves of different ages as predicted by a monomolecular function with horizontal shift

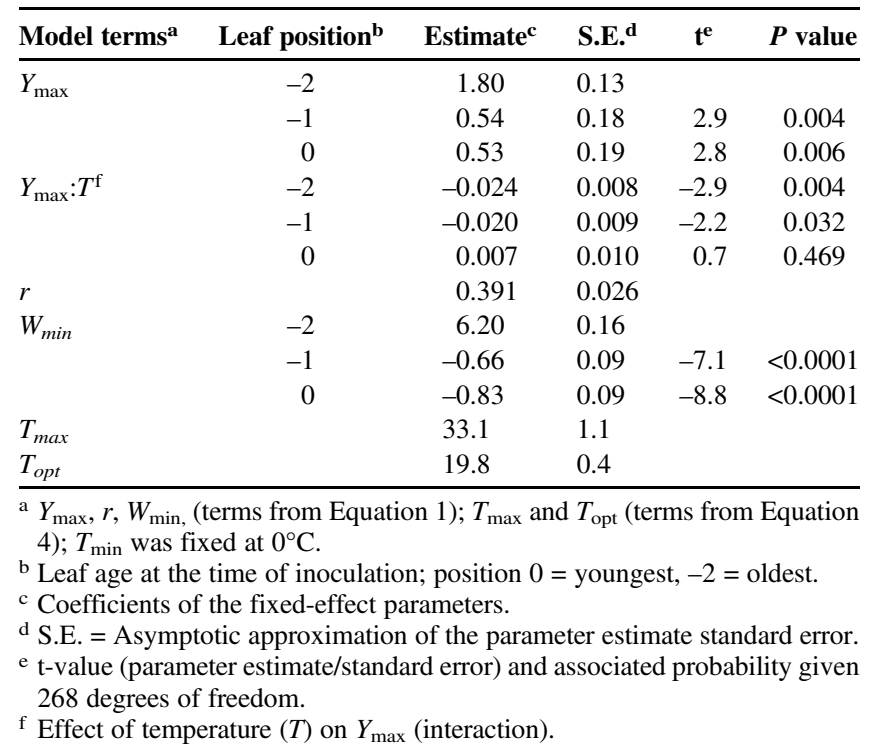

akin to a reduction in virulence (compatibility) than reducing aggressiveness of spores, since they are not slowed, but stopped (MacHardy 1996). Past studies have shown that spore germination, appressoria formation, and cuticle penetration are not different between young and older leaves of cultivars, either resistant or susceptible to apple scab (Valsangiacomo and Gessler 1988), and thus the apparent increased time for infection and subsequent reduction in lesion numbers occur because of events during stroma formation. Notwithstanding the leaf age effect, it is possible that the infection efficiency reduction observed with increasing infection temperature for older leaves is related to metabolism involved in ontogenic resistance, higher temperatures

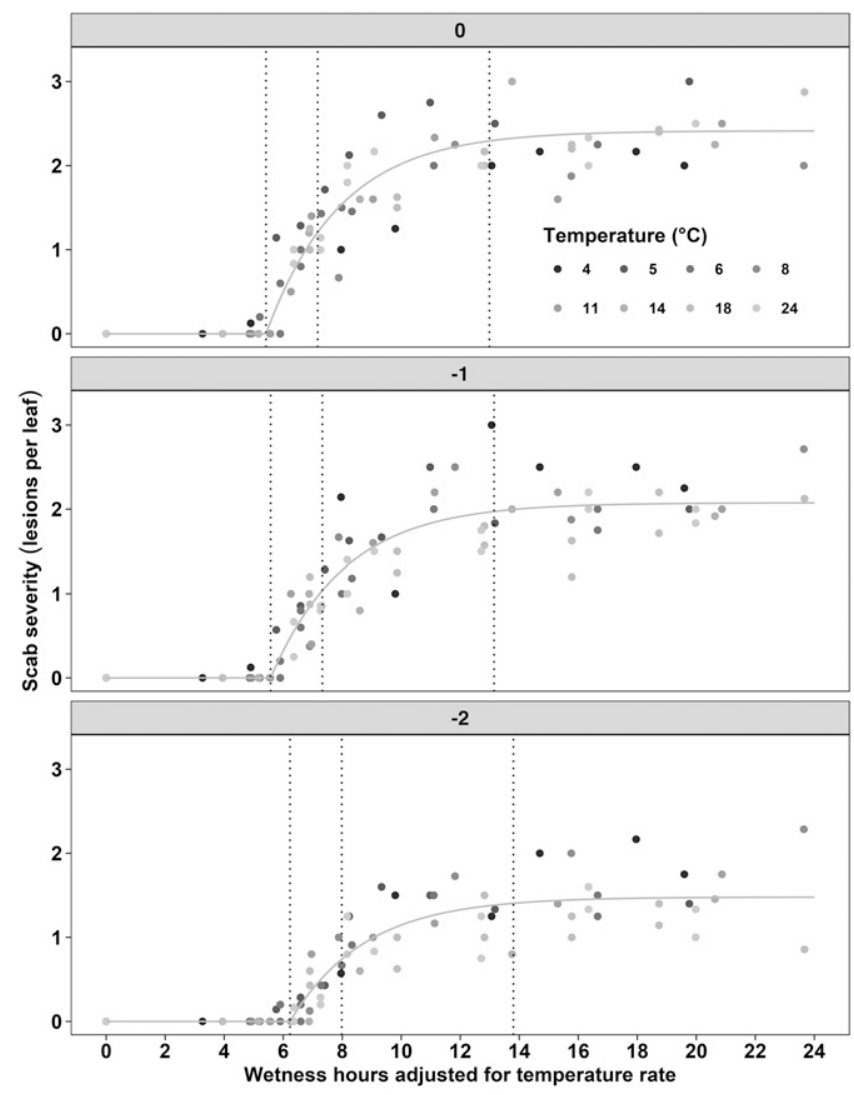

Fig. 3. Progression of ascospore infection of Venturia inaequalis corrected for increasing leaf age (panels $0,-1,-2$ ), in relation to wetness hours adjusted for temperature rate. Scab severity was modeled (solid lines) using Equation 1, whereas the temperature rate for wetness hours was modeled using Equation 4. The graph was truncated at $24 \mathrm{~h}$ to focus on the lower values. From left to right in each panel, vertical dotted lines represent the wetness time required for minimal, $50 \%$, and $95 \%$ spore infection, respectively.

Table 2. Effect of modifying the cardinal temperatures $\left(T_{\mathrm{opt}}, T_{\max }\right.$, and $\left.T_{\min }\right)$ of the temperature response rate function on the minimum $\left(W_{\min }\right)$ and $50 \%$ infection time ( $\left.W_{\text {adj }} 50 \%\right)$, the rate $(r)$ and overall fit of the Venturia inaequalis ascospore infection model (parameters as explained in Table 1)

\begin{tabular}{lcccccccc}
\hline $\begin{array}{l}\text { Model } \\
\text { variations }^{\mathbf{a}}\end{array}$ & $\boldsymbol{T}_{\mathbf{o p t}}$ & $\boldsymbol{T}_{\mathbf{m a x}}$ & $\boldsymbol{T}_{\mathbf{m i n}}$ & $\boldsymbol{W}_{\mathbf{m i n}}$ & $\boldsymbol{r}$ & $\begin{array}{c}\boldsymbol{W}_{\mathbf{a d j}} \\
\mathbf{5 0 \%}\end{array}$ & $\mathbf{C R M}^{\mathbf{b}}$ & $\mathbf{R M S E}^{\mathbf{c}}$ \\
\hline $\mathrm{m} .0$ & 19.8 & 33.1 & $0^{\mathrm{d}}$ & 5.4 & 0.39 & 7.1 & 0.0059 & 0.246 \\
m.c & 19.8 & 32.0 & -1 & 5.3 & 0.39 & 7.0 & 0.0059 & 0.246 \\
mOM.0 & $20.1^{\mathrm{d}}$ & $27.9^{\mathrm{d}}$ & $-15.6^{\mathrm{d}}$ & 4.6 & 0.42 & 6.2 & 0.0053 & 0.252 \\
mOM.50 & $20.1^{\mathrm{d}}$ & $34.8^{\mathrm{d}}$ & $0^{\mathrm{d}}$ & 5.5 & 0.35 & 7.5 & 0.0057 & 0.246 \\
m.18 & 20.1 & 28.1 & $-18^{\mathrm{d}}$ & 4.8 & 0.41 & 6.5 & 0.0049 & 0.251 \\
\hline
\end{tabular}

${ }^{a}$ For each model variation, only the cardinal temperatures used for the fit change. All calculations were done for the youngest unfurled leaf (Position 0).

${ }^{b} \mathrm{CRM}=$ coefficient of residual mass.

${ }^{\mathrm{c}}$ RMSE $=$ root mean square error.

${ }^{\mathrm{d}}$ Forced value; otherwise the temperatures were obtained from the model fit. 
resulting in quicker development of resistance. Conversely, it is possible that for temperatures below $5^{\circ} \mathrm{C}$, the lowest temperature for apple tree growth (Landsberg 1974), the metabolites responsible for ontogenic resistance are not produced, and infection can proceed more quickly. This may explain why the temperature response curve did not fit so well below $4^{\circ} \mathrm{C}$, as infection proceeded more quickly than what was expected by the optimal model. Forcing a minimum of $-18^{\circ} \mathrm{C}$ in the temperature rate equation resulted in an infection rate at $0^{\circ} \mathrm{C}$, about $0.1 \times$ the optimal rate at $20^{\circ} \mathrm{C}$ (for $T_{\max }$ at $28^{\circ} \mathrm{C}$ ) and did not introduce much bias if in fact the temperature relation is different below the host base temperature.

The optimum temperature of $20^{\circ} \mathrm{C}$ obtained for both minimum infection and 50\% infection rate is in line with the temperatures reported across Venturia spp. for both ascospores and conidia (GonzálezDomínguez et al. 2017; Keitt 1926; Magarey et al. 2005). However, if the Yin equation is extrapolated outside the temperature range we tested, the minimum $\left(0^{\circ} \mathrm{C}\right)$ and maximum infection temperature $\left(35^{\circ} \mathrm{C}\right)$ reported in the literature do not match our findings. Keitt (1926) and later Sys and Soenen (1970) reported that the maximum temperature at which infection was possible was $26^{\circ} \mathrm{C}$ and $26.5^{\circ} \mathrm{C}$, respectively, which more closely matches our modeled values. Similarly, for the minimum temperature some development occurs at $0^{\circ} \mathrm{C}$ to account for low temperature infections reported by Stensvand et al. (1997). Because of equipment limitations, we did not run experiments at temperatures below $4^{\circ} \mathrm{C}$, so our models were extrapolated to fit previously published data. Since variant "m.18" expands the scope of the model to all temperatures without consequence, we suggest it should be used.

Since each leaf was inoculated with approximately 25 ascospores, we could estimate the disease efficiency or lesion causing efficiency, meaning the proportion of inoculated spores to result in a visible lesion. Efficiency ranged from $9.7 \%$ for the youngest leaves to $5.9 \%$ for leaves at position -2 . Although the methodology was different, our results roughly match those found by Anagnostakis and Aylor (1991) who found $5.7 \%$ and $14 \%$ disease efficiency on shoots described as "stage 2" (4.6 unfurled leaves) and "stage 3" (5.3 unfurled leaves), respectively, for inoculum originating from cv. McIntosh leaves and inoculated on the same cultivar. We did not count the total number of leaves on each shoot at the time of inoculation, but all shoots would have been rated stage 2 or 3 with their system, since all had more than 4 leaves.

The different steps of infection of $V$. inaequalis were described in detail in previous studies, but less was done on the variability within the ascospore population to complete each step in the infection process. Our observed minimum infection time corresponded to the formation of the penetration pore and breaching of the epicuticular wax (Smereka et al. 1987). Whereas 50\% infection occurred in about $6.5 \mathrm{~h}$ in our study, $50 \%$ germination and appressoria formation was observed in about 9 and $14 \mathrm{~h}$, respectively, in the study of Turner et al. (1986). The lack of direct correspondence between appressoria formation and lesions most likely reflect that appressoria are not required for infection (Smereka et al. 1987) and underlines the necessity of directly modeling wetness time necessary for lesion formation and not derive it from appressoria formation as was done in the past for some simulation models. Similarly, relating the infection curve to previously published risk curves is not straightforward. The minimum risk curve of MacHardy and Gadoury (1989) and light infection curve of Schwabe (1980) fall between our $0.001 \%$ and $50 \%$ infection thresholds (Fig. 4). The light curve of Mills (1944) and moderate curve of Schwabe (1980) were close to the $75 \%$ infection mark in the present study. Finally, the moderate and severe infection levels of Mills (1944), respectively, were close to the $90 \%$ and $99 \%$ infection curves of our study, whereas the heavy curve of Schwabe (1980) falls between the $90 \%$ and $99 \%$ curves.

Since our approach purposely excluded ejection time and focused on infection, accounting for the ejection rate would be necessary for a full comparison, but this was beyond the scope of this work. Nonetheless, the contrast between the quick infection of spores once the minimum infection criteria are met in the present study and the more progressive Mills and Schwabe curves, suggests that the latter are mostly reflecting the slower progressive ejection of spores. Despite the shift in risk assessment between these, it is still remarkable that the curves are mostly parallel to our proposed curves over a wide temperature range.

Suggesting a 5-h minimal wetness threshold for infection at optimal temperatures may be perceived as an overly conservative step backward, but when interpreted in context, this may be a necessary adjustment. For the "average" leaf in the orchard, the minimum infection threshold is necessarily more than $5 \mathrm{~h}$, because only a fraction of scab susceptible leaves are the youngest. For unsprayed shoots, the probability of ascospores reaching and infecting the youngest most susceptible leaf is lower than for leaves slightly older, because the surface area of the older leaves is bigger. However, in commercial orchards where fungicides are applied during the time of rapid leaf expansion, only the youngest leaf is often left uncovered, and thus the shorter infection period requirement for the youngest leaf is important in practice. A revision of the Mills infection period explained how day versus night rain may have contributed to an over-estimation of the conditions required for infection in practice (MacHardy and Gadoury 1989). It is likely that the difference in infection time between younger and older leaves may have also contributed to this discrepancy.

Although it was expected that adjustments in simulation model assumptions would be needed to account for the observed difference in infection time between the fastest and the slowest spores, our results highlighted that the shape of the distribution currently used in models also needs adjustments. For instance, the leaf wetness time required to reach $50 \%$ infection (about $7 \mathrm{~h}$ at $20^{\circ} \mathrm{C}$ ) is substantially lower than what is currently implemented in the widely used RIMpro software (12 h). RIMpro also assumes the infection rate of ascospores landed on leaf tissue is normally distributed in the population, with a 3-h standard deviation, and thus the $5 \%$ infection time is 2 standard deviations lower than the mean (ex: $6 \mathrm{~h}$ at $20^{\circ} \mathrm{C}$ ). Similarly, $95 \%$ infection is predicted after $18 \mathrm{~h}$, derived from data published by Moore (1964). This last value is close to our estimate of complete infection $(95 \%$ at $13 \mathrm{~h}$;

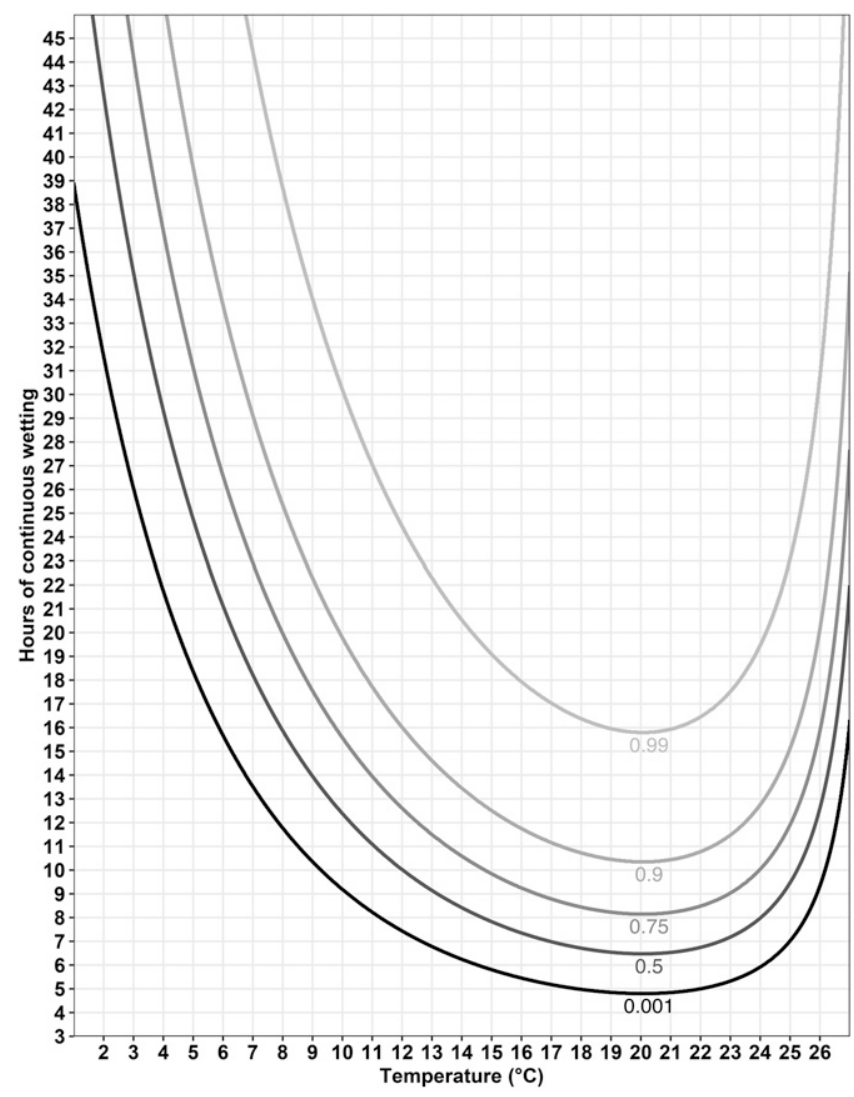

Fig. 4. Proportion of ascospores of Venturia inaequalis resulting in scab lesions on the youngest unfurled leaf in relation to wetness duration and temperature during infection. The 0.001 infection threshold was arbitrarily chosen to illustrate a minimum infection threshold. The graph styling is an homage to W. D. Mills. 
$99 \%$ at $16 \mathrm{~h}$ ). It follows that scab severity progresses more quickly than anticipated by RIMpro in the first hours after the infection threshold is reached, and slower as the last spores infect the host.

The whole dynamic of spore maturation, ejection, and infection is more easily represented by mechanistic models (Magarey et al. 2005), such as RIMpro, which link each of these processes and efficiently account for the effect of daylight, varying temperature, etc. The advantage of using mechanistic models is that we can implement all this information as a chain of biological processes that work on a population, and decisions can be made based on quantitative infection values reached at a certain stage in time. In this spirit, the data provided in this study are meant to improve the infection submodel of such models.

In practice, the necessity to spray does not depend on the faith of the first spores that are ejected with the first drops of rain, but by the spore quantity distributed over time and their development. The lesion distribution resulting from several hours of rain is always the result of overlapping development curves of all spore cohorts ejected over time and their quantity. When interpreted alongside the gradual ejection, a better understanding of the ascospore infection dynamics has practical applications, as it helps better assess the proportion of spores killed prior to fungicide wash-off and time sprays that have limited postinfection properties. For instance, fungicides that lack rain fastness but that are either less toxic to the environment and/or admissible in organic production can be used at the optimal time. A good example of this is potassium bicarbonate (Jamar et al. 2008; Philion and Joubert 2015; Trapman 2008), which must be sprayed in the early stages of infection. Another practical consequence of our findings is that since infections proceed more quickly than anticipated in the first hours following the minimal infection threshold, any underestimating errors in wetness time can lead to more risk than anticipated otherwise. Although improvements may be minor, they incrementally build confidence from consultants and users toward DSSs.

There is general agreement that the Light infection curve of Mills (1944) overestimated the number of wetness hours required for a measurable scab risk in some orchards, but blindly substituting this with a minimal risk infection curve is also known to result in unnecessary sprays in most orchards. Although laboratory studies provide a more accurate assessment of the hours required for infection compared with empirical studies (MacHardy and Gadoury 1989), it is also true that simple infection curves cannot be used without the other components of risk for optimal spray advisory. In orchards with abundant inoculum of ascospores of $V$. inaequalis ready for discharge, for rains occurring during daylight hours and when the temperature does not delay ejection, the minimum infection curve may be the required application threshold on very susceptible cultivars. Conversely, including knowledge of inoculum level (MacHardy et al. 1993), cultivar susceptibility, proper accounting of spore maturation, discharge and infection rate, makes it possible to optimize the number of sprays needed for apple scab control. If only the early onset and steepness of the infection curve proposed in this study are implemented in DSSs, an increased number and more severe infection events will be reported without any relation to actual risk or need to spray.

It has been known since the pioneering work of Keitt and Jones (1926) that primary infection of apple scab is possible in as few as $5 \mathrm{~h}$ of wetness at optimal temperatures. Although this is not common, this possibility should be recognized in population-based dynamic decision support systems and properly accounted for. Conversely, our findings should not be implemented in simple DSSs that only consider infection requirements, as it would be counterproductive and lead to unnecessary sprays. Now that many aspects of apple scab epidemiology are well understood, modern mechanistic DSSs can become much more precise and useful for tailored spray recommendations than the empirical Mills table ever could.

\section{Acknowledgments}

We thank the staff at IRDA and the many summer students that worked very hard to make this project possible.

\section{Literature Cited}

Anagnostakis, S. L., and Aylor, D. E. 1991. Efficiency of ascospores of Venturia inaequalis in producing scab lesions on apple leaves. Plant Dis. 75:918-920.

Arauz, L. F., and Sutton, T. B. 1989. Temperature and wetness duration requirements for apple infection by Botryosphaeria obtusa. Phytopathology 79:440-444.

Archontoulis, S. V., and Miguez, F. E. 2015. Nonlinear regression models and applications in agricultural research. Agron. J. 107:786-798.

Baty, F., Ritz, C., Charles, S., Brutsche, M., Flandrois, J.-P., and DelignetteMuller, M.-L. 2015. A toolbox for nonlinear regression in R: The package nlstools. J. Stat. Softw. 66:1-21.

Bolker, B. M. 2008. Ecological models and data in R. Princeton University Press, Princeton, NJ.

Brière, J.-F., Pracros, P., Le Roux, A.-Y., and Pierre, J.-S. 1999. A novel rate model of temperature-dependent development for arthropods. Environ. Entomol. 28:22-29.

Brook, P. J. 1969. Effects of light, temperature, and moisture on release of ascospores by Venturia inaequalis (Cke.). Wint. N.Z. J. Agric. Res. 12:214-227.

Brown, R. F., and Mayer, D. G. 1988. Representing cumulative germination. 2 The use of the Weibull function and other empirically derived curves. Ann. Bot. 61:127-138.

Butler, D. R., and Jadhav, D. R. 1991. Requirements of leaf wetness and temperature for infection of groundnut by rust. Plant Pathol. 40:395-400.

Butler, D. R., Wadia, K. D. R., and Jadhav, D. R. 1994. Effects of leaf wetness and temperature on late leaf-spot infection of groundnut. Plant Pathol. 43: 112-120.

Damos, P., and Savopoulou-Soultani, M. 2012. Temperature-driven models for insect development and vital thermal requirements. Psyche (Stuttg.) 2012: 123405 .

Duthie, J. A. 1997. Models of the response of foliar parasites to the combined effects of temperature and duration of wetness. Phytopathology 87:1088-1095.

Gadoury, D. M., and MacHardy, W. E. 1982. A model to estimate the maturity of ascospores of Venturia inaequalis. Phytopathology 72:901-904.

Gadoury, D. M., Seem, R. C., Stensvand, A., and Falk, S. P. 1996. Minimum criteria for infection by Venturia inaequalis, and severity of scab at different phenological stages of apple. Phytopathology 86:S122.

Gadoury, D. M., Stensvand, A., and Seem, R. C. 1998. Influence of light, relative humidity, and maturity of populations on discharge of ascospores of Venturia inaequalis. Phytopathology 88:902-909.

Gilpatrick, J. D., Smith, C. A., and Blowers, D. R. 1972. A method of collecting ascospores of Venturia inaequalis for spore germination studies. Plant Dis. Rep. 56:39-42.

González-Domínguez, E., Armengol, J., and Rossi, V. 2017. Biology and epidemiology of Venturia species affecting fruit crops: A review. Front. Plant Sci. 8:1496 https://www.frontiersin.org/articles/10.3389/fpls.2017.01496/full.

Jamar, L., Lefrancq, B., Fassotte, C., and Lateur, M. 2008. A during-infection spray strategy using sulphur compounds, copper, silicon and a new formulation of potassium bicarbonate for primary scab control in organic apple production. Eur. J. Plant Pathol. 122:481-493.

James, J. R., and Sutton, T. B. 1982. Environmental factors influencing pseudothecial development and ascospore maturation of Venturia inaequalis. Phytopathology 72:1073-1080.

Keitt, G. W. 1926. Some relations of environment to the epidemiology and control of apple scab. Proc. Nat. Acad. Sci. 12:68-74.

Keitt, G. W., and Jones, L. K. 1926. Studies of the epidemiology and control of apple scab. Wis. Agric. Exp. Stn. Bull. 73:194 pp.

Kozak, M., and Piepho, H.-P. 2018. What's normal anyway? Residual plots are more telling than significance tests when checking ANOVA assumptions. J. Agron. Crop Sci. 204:86-98.

Lactin, D. J., Holliday, N. J., Johnson, D. L., and Craigen, R. 1995. Improved rate model of temperature-dependent development by arthropods. Environ. Entomol 24:68-75.

Lalancette, N., Ellis, M. A., and Madden, L. V. 1988. Development of an infection efficiency model for Plasmopara viticola on American grape based on temperature and duration of leaf wetness. Phytopathology 78:794-800.

Landsberg, J. J. 1974. Apple fruit bud development and growth; analysis and an empirical model. Ann. Bot. 38:1013-1023.

MacHardy, W. E. 1996. Apple Scab: Biology, Epidemiology, and Management. The American Phytopathology Society, St. Paul, MN.

MacHardy, W. E., and Gadoury, D. M. 1989. A revision of Mills's criteria for predicting apple scab infection periods. Phytopathology 79:304-310.

MacHardy, W. E., Gadoury, D. M., and Rosenberger, D. A. 1993. Delaying the onset of fungicide programs for control of apple scab in orchards with low potential ascospore dose of Venturia inaequalis. Plant Dis. 77:372-375.

Madden, L. V., Hughes, G., and van den Bosch, F. 2007. The Study of Plant Disease Epidemics. American Phytopathological Society, St. Paul, MN.

Magarey, R. D., Sutton, T. B., and Thayer, C. L. 2005. A simple generic infection model for foliar fungal plant pathogens. Phytopathology 95:92-100.

Mills, W. D. 1944. Efficient use of sulfur dusts and sprays during rain to control apple scab. Cornell Ext. Bull. 630:1-4.

Moore, M. H. 1964. Glasshouse experiments on apple scab: I. Foliage infection in relation to wet and dry periods. Ann. Appl. Biol. 53:423-435. 
Nash, J. E., and Sutcliffe, J. V. 1970. River flow forecasting through conceptual models part I-A discussion of principles. J. Hydrol. (Amst.) 10:282-290.

Olivier, J. M., Lambert, C., and Lefeuvre, M. 1983. Application du thermohumectographe KIT-INRA. Etude des risques de tavelure du pommier à l'échelle du Maine-et-Loire (France). EPPO Bull. 13:47-56.

Philion, V., and Joubert, V. 2015. Use pattern and limits of potassium bicarbonate for apple scab control in Quebec orchards. IOBC WPRS Bull. 110:199-212.

Pinheiro, J., and Bates, D. 2004. Mixed-Effects Models in S and S-PLUS. Springer, New York.

Pinheiro, J., Bates, D., DebRoy, S., Sarkar, D., and R Core Team. 2018. nlme: Linear and nonlinear mixed effects models. Available at: https://cran.rproject.org/web/packages/nlme/index.html.

Preece, T. F. 1964. Continuous testing for scab infection weather using apple rootstocks. Plant Pathol. 13:6-9.

R Core Team. 2018. R: A Language and Environment for Statistical Computing. R Foundation for Statistical Computing, Vienna, Austria. Available at: https:// www.R-project.org/.

Rossi, V., Giosuè, S., and Bugiani, R. 2007. A-scab (Apple-scab), a simulation model for estimating risk of Venturia inaequalis primary infections. EPPO Bull. 37:300-308.

Scherm, H., and van Bruggen, A. C. H. 1994. Effects of fluctuating temperatures on the latent period of lettuce downy mildew (Bremia lactucae). Phytopathology 84 : 853-859.

Schwabe, W. F. S. 1980. Wetting and temperature requirements for apple leaf infection by Venturia inaequalis in South Africa. Phytophylactica 12: $69-80$.

Smereka, K. J., Machardy, W. E., and Kausch, A. P. 1987. Cellular differentiation in Venturia inaequalis ascospores during germination and penetration of apple leaves. Can. J. Bot. 65:2549-2561.

Spiess, A.-N. 2018. propagate: Propagation of Uncertainty. Available at: https:// cran.r-project.org/web/packages/propagate/index.html.
Stensvand, A., Gadoury, D. M., Amundsen, T., Semb, L., and Seem, R. C. 1997. Ascospore release and infection of apple leaves by conidia and ascospores of Venturia inaequalis at low temperatures. Phytopathology 87:1046-1053.

Sys, S., and Soenen, A. 1970. Investigations on the infection criteria of scab (Venturia inaequalis Cooke. Wint.) on apples with respect to the table of Mills and Laplante. Agric. Louvain. 18:3-8.

Tosic, M., and Vasic, V. 1977. Prilog proucavanju primene aparata P. 1983/74 u prognozi cadjave krastavosti jabuke. Prethodno saopstenje. Zast. Bilja 28:167-172.

Trapman, M. 2008. Practical experience with the use of baking powder (potassium bicarbonate) for the control of apple scab (Venturia inaequalis). Pages 68-75 in: Ecofruit: 13th International Conference on Cultivation Technique and Phytopathological Problems in Organic Fruit-Growing, Weinsberg/Germany. Available at: http://orgprints.org/13646/1/068-075.pdf.

Trapman, M., and Polfliet, M. 1997. Management of primary infections of applescab with the simulation program RIMpro: Review of four years field trials. IOBC WPRS Bull. 20(9):241-250.

Turner, M. L., MacHardy, W. E., and Gadoury, D. M. 1986. Germination and appressorium formation by Venturia inaequalis during infection of apple seedling leaves. Plant Dis. 70:658-661.

Valsangiacomo, C., and Gessler, C. 1988. Role of the cuticular membrane in ontogenic and Vf-resistance of apple leaves against Venturia inaequalis. Phytopathology 78:1066-1069.

Webb, D. H., and Nutter, F. W., Jr. 1997. Effects of leaf wetness duration and temperature on infection efficiency, latent period, and rate of pustule appearance of rust in alfalfa. Phytopathology 87:946-950.

Wickham, H. 2017. Tidyverse: Easily install and load the "tidyverse." Available at: https://cran.r-project.org/web/packages/tidyverse/index.html.

Yan, W., and Hunt, L. A. 1999. An equation for modelling the temperature response of plants using only the cardinal temperatures. Ann. Bot. 84:607-614

Yin, X., Kropff, M. J., McLaren, G., and Visperas, R. M. 1995. A nonlinear model for crop development as a function of temperature. Agric. For. Meteorol. 77:1-16. 